\title{
Hypogonadism: Easy to define, hard to diagnose, and controversial to treat
}

\author{
Joshua Sterling, MD; ${ }^{*}$ Aaron M. Bernie, $M D ;{ }^{\dagger}$ Ranjith Ramasamy, $M D^{\S}$
}

*New York Medical College, Valhalla NY; †Department of Urology, New York Presbyterian Hospital, Weill Cornell Medical College, New York, NY; §Department of Urology, Baylor College of Medicine, Houston, TX

Cite as: Can Urol Assoc J 2015;9(1-2):65-8. http://dx.doi.org/10.5489/cuaj.2416

Published online February 5, 2015.

\section{Introduction}

It has been estimated that hypogonadism currently affects up to 4 million American men, and as the population ages, this number will only rise. Nevertheless, only $5 \%$ of these men are being treated actively for hypogonadism. ${ }^{1}$ According to the most recent United States census, $13 \%$ of the population is above the age of 65 and this is expected to increase by $16 \%$ by $2020 .^{2}$

The Endocrine Society's 2010 clinical practice guidelines ${ }^{3}$ outline a protocol for evaluating an adult male patient suspected of having androgen deficiency. In contrast to menopause, in which females experience all of the symptoms associated with the loss of estrogen within a well-defined period, men undergo a slow and gradual decline in their testosterone levels with a seemingly insidious onset of symptoms. ${ }^{3}$ For patients with a history and physical examination consistent with hypogonadism, the diagnosis can be "easily" confirmed by measuring serum testosterone levels, but how to interpret the results and decide who should be treated remains less clear.

Hypogonadism itself is broadly referred to as testicular failure associated with androgen deficiency, ${ }_{1}^{4}$ although such a broad definition encompasses many men and has required sub-classification based on cause and age. ${ }^{5}$ Late-onset hypogonadism $(\mathrm{LOH})$ has been defined as a complex cluster of symptoms seen in aging men accompanied by a decrease in serum testosterone levels. ${ }^{6}$ Despite these definitions, the identification of these men is complicated and difficult.

Currently, one of the most popular methods for hypogonadism screening is the use of questionnaires, such as The Saint Louis University Androgen Deficiency in the Aging Male (ADAM) questionnaire, ${ }^{7}$ The Aging Male's Symptoms
(AMS) rating scale, ${ }^{8}$ the Massachusetts Male Aging Study (MMAS) questionnaire, ${ }^{9}$ and the New England Research Institutes (NERI) hypogonadism questionnaire. ${ }^{10}$ Like the many other screening tools, they have mixed success in their ability to predict the presence of hypogonadism, with sensitivity that can range from $59 \%$ to $97 \%$ and specificity that can range from $19 \%$ to $59 \% .^{9,11-16}$ Despite these wide ranges, they provide a quick and non-invasive way to screen for hypogonadism, but a low testosterone level remains the gold standard in diagnosis.

\section{Etiology of hypogonadism}

For men with confirmed low testosterone levels, serum luteinizing hormone $(\mathrm{LH})$ and follicle-stimulating hormone (FSH) levels should be measured to determine whether the cause is of primary or secondary origin. Low testosterone with high $\mathrm{LH} / \mathrm{FSH}$ levels suggest a primary etiology, which includes age-related hypogonadism, Klinefelter's syndrome, uncorrected cryptorchidism, cancer chemotherapy, radiation therapy, trauma, or a history of mumps orchitis or orchiectomy. Some of the commonly used medications can also lead to low testosterone. For example, opioids and anabolic steroids are some of the common offenders. Low testosterone combined with low or normal LH/FSH levels suggests a secondary etiology of hypogonadism, which includes pituitary neoplasms, hyperprolactinemia, hemochromatosis infiltrative disorders, genetic disorders of $\mathrm{GnRH}$ secretion, genetic disorders of gonadotropin secretion or action, genetic disorders of pituitary development, eating disorders, or use of anabolic steroids. ${ }^{17}$ It is hard to know what percent of patients diagnosed with hypogonadism are affected by a primary condition and what percent are affected by a secondary condition because most patients diagnosed with $\mathrm{LOH}$ have a mixed disease. However, the most clinically relevant primary diseases, Klinefelter's and testicular tumours, affect 1 in 500 men and 12 in 10000 men, respectively. 
Sterling et al.

The most relevant forms of secondary hypogonadism are prolactinemia, which has several etiologies and thus a variable prevalence, and Kallmann syndrome, which affects 1 in $10000 .^{18}$

The effect of chronic conditions, such as aging, diabetes mellitus, metabolic syndrome, and malignancy on testosterone levels cannot be ignored. The European Male Aging Study (EMAS) looked at over 3000 men ages 40 to 79 to see how age and other comorbidities influence the hypothalamic-pituitary-thyroid (HPT) axis. The EMAS study looked at serum testosterone and LH levels of participants across 4 age groups: $40-49,50-59,60-69,70-79 .{ }^{19}$ The largest trend was seen with free testosterone (FT), which showed a drop of $1.3 \%$ per year. Another significant trend was seen with regards to testosterone and patients' weight as determined by body mass index (BMI). Both overweight (BMI 25-30) and obese men $(\mathrm{BMI}>30)$ had total testosterone $(\mathrm{TT})$ that was 2.32 and $5.09 \mathrm{nmol} / \mathrm{L}$ lower, respectively, than the non-obese group. Similarly, the average FT was $17.6 \mathrm{pmol} / \mathrm{L}$ lower in overweight men when compared to non-obese men and $53.72 \mathrm{pmol} / \mathrm{L}$ lower in obese men compared to nonobese men. ${ }^{19}$ Another subset of the MMAS looked at men without obesity, alcoholism, prescription medications, prostate problems, cancer, diabetes, and coronary heart disease, and compared them with men who reported one or more of the above conditions. Levels of testosterone and sex hormone binding globulin (SHBG) in the group with at least 1 comorbidity were typically $10 \%$ to $15 \%$ lower across all age ranges. However, further analysis suggested that obesity may have been responsible for much of this difference in each of these groups. ${ }^{12}$

\section{Challenges with diagnosis of hypogonadism}

While the 2010 Endocrine Society Guidelines provide a good starting point for determining diagnosis and cause of hypogonadism, they may oversimplify this complex disorder. For example, the current guidelines use $300 \mathrm{ng} / \mathrm{dL}$ as the threshold for diagnosing low testosterone. But can this threshold really be applied to every man evaluated? This level was set as the lower limit for normal serum testosterone because men were more symptomatic below this threshold than above it. ${ }^{20}$ In other words, hypogonadal symptoms define where the lower limit of normal is set, and the most common symptoms at $300 \mathrm{ng} / \mathrm{dL}$ are decreased frequency of sexual thoughts, decreased sexual desire, and erectile dysfunction. Several studies have shown that while there are no serum testosterone levels universally associated with specific hypogonadal symptoms, the serum testosterone levels at which an individual will experience symptoms lie within a small range. ${ }^{21}$ With that in mind, a stricter definition of hypogonadism, which includes the presence of at least 3 sexual symptoms as well as a low total and free testoster- one, has been suggested. ${ }^{5}$ This person-to-person variability makes identifying at-risk individuals extremely difficult, forcing physicians to rely more on symptoms reported by patients than on results of specific blood test values to make a diagnosis.

Serum testosterone levels fluctuate over the course of the day as well as from day-to-day, typically peaking in the early morning and slowly declining through the course of the day until they reach their nadir in the evening. Additionally, measurements of testosterone from the same sample often vary depending on which laboratory processes the sample, even if the laboratories use the same protocols. It is recommended that serum testosterone levels be measured in the morning, but this approach has been challenged, and often is not the norm in clinical practice. ${ }^{22}$ Moreover, while 2 low readings are used to diagnose hypogonadism, up to $15 \%$ of healthy men may have a low testosterone reading over any 24-hour period. ${ }^{23}$

The need for a confirmatory testosterone measurement is complicated by a lack of standardization in the methods used to measure testosterone, as well as by the lack of precision and accuracy in the commonly used immunoassay platforms. ${ }^{22}$ The method of choice for TT is liquid chromatography-tandem mass spectrometry (LC-MS/MS). However, this methodology is available to a limited number of hospitals and office practices, despite its acceptance as the gold standard, because of its cost and the requirement of the presence of trained personnel. Despite LC-MS/ MS being the gold standard, enzyme immunoassays and other similar tests are the most commonly used methods to measure testosterone in non-reference hospitals and commercial laboratories. These alternate testing platforms have adequate accuracy for eugonadal men, but not for men with TT levels less than $300 \mathrm{ng} / \mathrm{dL}$ as compared to LC-MS. ${ }^{22}$ In addition, when a standard $299 \mathrm{ng} / \mathrm{dL}$ sample was used, a range of 198 to $364 \mathrm{ng} / \mathrm{dL}$ was reported by 8 different labs around the United States, a variation of $-33 \%$ and $+21 \% .{ }^{24}$ This range of over $50 \%$ variability is significantly more than the $10 \%$ intra-individual variability that is acceptable for most laboratory tests, showing that even the best practices are unreliable in diagnosing hypogonadism.

TT is the sum of the FT in the blood and the testosterone bound to SHBG and albumin. Because only $2 \%$ to $3 \%$ of the TT is free and available for the body to use, measuring FT might be a better way to assess hypogonadism. Normally, FT ranges from 4 to $8 \mathrm{ng} / \mathrm{mL} .{ }^{5}$ Because of this small range, FT levels are very sensitive to changes in SHBG, making it important to be aware of how certain conditions affect SHBG in the body. Factors that increase SHBG include aging, hyperthyroidism, estrogens, HIV, anticonvulsants, cirrhosis, and hepatitis. Factors that decrease SHBG include obesity (BMI >30), diabetes mellitus, hypothyroidism, glucocorticoids, androgenic steroids, nephrotic syndromes, and acro- 
megaly. ${ }^{25}$ These conditions can cause a significant change in calculated FT levels, which can lead to an incorrect or missed diagnosis of hypogonadism. Thus, when a patient is complaining of hypogonadism-related symptoms but his TT levels are within the near normal range, the next step should be to check the FT and/or SHBG levels to determine whether the changes might be responsible for the symptoms.

\section{Controversies in treatment of hypogonadism}

It remains unclear as to who, if anyone, should be treated for hypogonadism. What modality of testosterone supplementation therapy (TST) is best? What should target testosterone levels be? How long should TST be continued? The goal of TST is to reduce symptoms and increase the general quality of life, while inducing minimal adverse side effects. Previously, normal adult males who have developed hypogonadism reported marked improvement in libido, energy, and sexual function while receiving TST. Men receiving TST have reported improvement in mood, energy, memory, increases in fat-free body mass by $5 \%$, strength by $45 \%$ and bone density by $40 \%$ with a decrease in whole body fat mass. ${ }^{26,27} \mathrm{~A}$ failure to report improvement after the serum testosterone has returned to normal levels could suggest another underlying cause for the symptoms, such as natural process of aging or comorbidities.

All of these benefits of TST have led to an increased quality of life in men, but TST is not without potential adverse effects. The widespread use of TST has been impeded by concerns over the potential side effects, the most serious being increased risk of cardiovascular disease and adenocarcinoma of the prostate, and lesser side effects including worsening sleep apnea, erythrocytosis and venous thromboembolism. Most early stage adenocarcinomas of the prostate are androgen dependent, and the large majority of early adenocarcinomas are clinically silent. However, this valid concern should not prevent a patient from receiving the benefits of TST as long as prostate cancer screening is done before therapy is initiated and the patient is closely monitored throughout treatment. ${ }^{26}$

With regards to TST and its relation to increased cardiovascular events, several recent studies have highlighted the main proposed mechanisms and concerns. Vigen and colleagues suggest that the administration of testosterone could increase platelet activation and thus lead to the formation of coronary plaques, or that a testosterone metabolite may result in an increased activation of monocytes within the endothelium which leads to atherosclerotic plaques. ${ }^{27}$ In their study of men who had recently undergone coronary angiography, they found an association between TST and allcause mortality, myocardial infarction (MI) and stroke, but this study is not without flaws. Initial absolute numbers from this study suggested that there was an increase in non-fatal
MIs in men who did not get TST, but the addition of multiple statistical analyses reversed these results. In addition, this study included only men undergoing coronary angiogram and thus lacked any randomization.

The Testosterone in Older Men with Mobility Limitations (TOM) trial $^{28}$ and the Finkle and colleagues' retrospective study $^{29}$ are 2 additional studies that reported an increased risk of cardiovascular events with TST. Similarly to Vigen's study, there were several flaws with these studies. Finkle and colleagues looked at insurance billing claims to see if the rate of MI increased after TST. They found an increase in the rate of $\mathrm{MI}$ in men post-TST prescription when compared to the $\mathrm{MI}$ rate pre-prescription, as well as a higher rate of $\mathrm{MI}$ in men prescribed TST compared with men who were prescribed phosphodiesterase type- 5 inhibitors. However, the overall design of this study has its limitations. It was also uncontrolled, and the investigators did not report why patients were prescribed TST, what dosages were given, or what other cardiovascular risk factors patients had. ${ }^{29}$ Finally, this study looked at the incidence of $\mathrm{Ml}$ in the first 90 days after starting treatment, and it is highly unlikely that TST could have produced physiological changes resulting in an MI that quickly.

The TOM study had a better experimental design but again had several major flaws. This trial was a randomized, placebo-controlled, double-blind trial, but the trial lacked power. The drug arm of the TOM trial had significantly more adverse events compared to the placebo arm (22 compared to 5); with such small numbers, it is possible that the difference between the groups may have been due to chance. ${ }^{28}$ Additionally, a further analysis of the reported events found that the diversity of these events renders them less susceptible to a single inciting mechanism. Because of the limitations in all of the available studies, the FDA has not concluded that TST increases the risk of stroke, MI, or mortality.

As suggested by the studies discussed above, one of the main concerns with TST is the lack of randomized controlled trials regarding therapy, as well as guidelines on how to follow men undergoing TST. This was another limitation of the TOM trial, where the participants all began the trial with TT levels between 100 and $300 \mathrm{ng} / \mathrm{dL}$ with a goal to increase testosterone to 500 and $1000 \mathrm{ng} / \mathrm{dL}$ without consideration of starting point, severity of symptoms, or other comorbidities. ${ }^{28}$ The investigators do not mention if the participants who were outside the target testosterone range exhibited different improvements compared with the participants that were within the target testosterone range. In addition, there are no set standards regarding duration of TST or when therapy should be discontinued. While this type of data is readily available for many other widely used therapies in the medical field, studies of TST that would allow for standardization of therapy do not exist. 


\section{Conclusion}

Despite attempts to standardize the diagnosis and treatment of hypogonadism, the process is not straightforward. The variability associated with the laboratory diagnosis of low testosterone, the multiple comorbid conditions and aging process that can mimic symptoms, and the challenge of balancing treatment with side effects are just a few of the issues that practitioners face when treating men with suspected hypogonadism. While the last decade has seen an increase in both prevalence and awareness of hypogonadism, the balance between properly diagnosing men who are suffering from the effects of low testosterone and identifying the appropriate candidates for treatment remains elusive.

Competing interests: Authors declare no competing financial or personal interests.

This paper has been peer-reviewed.

\section{References}

1. Seftel A. Male hypogonadism. Part II: Etiology, pathophysiology, and diagnosis. Int I Impot Res 2006;18:223-8. http://dx.doi.org/10.1038/si.iij.3901365

2. Werner CA. The Older Population: 2010. United States Census Bureau; 2010.

3. Bhasin $S$, Cunningham GR, Hayes FJ, et al. Testosterone therapy in men with androgen deficiency syndromes: An Endocrine Society clinical practice guideline. J Clin Endocrinol Metab 2010;95:2536-59. http://dx.doi.org/10.1210/ic.2009-2354

4. Rey RA, Grinspon RP, Gottlieb S, et al. Male hypogonadism: An extended classification based on a developmental, endocrine physiology-based approach. Andrology 2013;1:3-16. http://dx.doi.org/10.1111/ j.2047-2927.2012.00008.x

5. Wu FC, Tajar A, Beynon JM, et al. Identification of late-onset hypogonadism in middle-aged and elderly men. N Engl J Med 2010;363:123-35. http://dx.doi.org/10.1056/NEJMoa0911101

6. Gray A, Feldman HA, McKinlay JB, et al. Age, disease, and changing sex hormone levels in middle-aged men: Results of the Massachusetts Male Aging Study. J Clin Endocrinol Metab 1991;73:1016-25. http:// dx.doi.org/10.1210/icem-73-5-1016

7. Morley JE, Charlton E, Patrick $P$, et al. Validation of a screening questionnaire for androgen deficiency in aging males. Metabolism 2000;49:1239-42. http://dx.doi.org/10.1053/meta.2000.8625

8. Badia $X$, Herdman $M$. The importance of health-related quality-of-life data in determining the value of drug therapy. Clin Ther 2001;23:168-75. http://dx.doi.org/10.1016/50149-2918(01)80039-4

9. Smith KW, Feldman HA, McKinlay JB. Construction and field validation of a self-administered screener for testosterone deficiency (hypogonadism) in ageing men. Clin Endocrinol (0xf) 2000;53:703-11. http:// dx.doi.org/10.1046/i.1365-2265.2000.01152.x

10. Rosen RC, Araujo AB, Connor MK, et al: Assessing symptoms of hypogonadism by self-administered questionnaire: qualitative findings in patients and controls. Aging Male 2009;12:77-85. http://dx.doi. org/10.1080/13685530903184043

11. Blumel JE, Chedraui P, Gili SA, et al. Is the Androgen Deficiency of Aging Men (ADAM) questionnaire useful for the screening of partial androgenic deficiency of aging men? Maturitas 2009;63:365-8. http:// dx.doi.org/10.1016/i.maturitas.2009.04.004
12. Cabral RD, Busin L, Rosito TE, et al. Performance of Massachusetts Male Aging Study (MMAS) and androgen deficiency in the aging male (ADAM) questionnaires in the prediction of free testosterone in patients aged 40 years or older treated in outpatient regimen. Aging Male 2014;17:147-54. http:// dx.doi.org/10.3109/13685538.2014.908460

13. Heinemann LA, Moore C, Dinger JC, et al. Sensitivity as outcome measure of androgen replacement: The AMS scale. Health Qual Life Outcomes 2006;4:23. http://dx.doi.org/10.1186/1477-7525-4-23

14. Martinez-Jabaloyas JM, Queipo-Zaragoza A, Rodriguez-Navarro R, et al. Relationship between the Saint Louis University ADAM questionnaire and sexual hormonal levels in a male outpatient population over 50 years of age. Eur Urol 2007;52:1760-7. http://dx.doi.org/10.1016/i.eururo.2007.05.021

15. Moore C, Huebler D, Zimmermann T, et al. The Aging Males' Symptoms scale (AMS) as outcome measure for treatment of androgen deficiency. Eur Urol 2004;46:80-7. http://dx.doi.org/10.1016/i. eururo.2004.01.009

16. Morley JE, Perry HM, 3rd, Kevorkian RT, et al. Comparison of screening questionnaires for the diagnosis of hypogonadism. Maturitas 2006;53:424-9. http://dx.doi.org/10.1016/i.maturitas.2005.07.004

17. Bhasin S, Basaria S. Diagnosis and treatment of hypogonadism in men. Best Pract Res Clin Endocrinol Metab 2011;25:251-70. http://dx.doi.org/10.1016/i.beem.2010.12.002

18. Dohle GR AS, Bettocchi C, Klesch $S$, et al. Guidelines on male hypogonadism. Arnhem, European Association of Urology; 2012. http://www.uroweb.org/gls/pockets/english/15_Male_Hypogonadism.pdf. Accessed January 26, 2015.

19. Wu FC, Tajar A, Pye SR, et al. Hypothalamic-pituitary-testicular axis dissuptions in older men are differentially linked to age and modifiable risk factors: The European Male Aging Study. J Clin Endocrinol Metab 2008;93:2737-45. http://dx.doi.org/10.1210/ic.2007-1972

20. Bhasin S, Cunningham GR, Hayes FJ, et al. Testosterone therapy in adult men with androgen deficiency syndromes: An endocrine society clinical practice guideline. J Clin Endocrinol Metab 2006;91:1995-2010. http://dx.doi.org/10.1210/ic.2005-2847

21. Kelleher $\mathrm{S}$, Conway AJ, Handelsman DJ. Blood testosterone threshold for androgen deficiency symptoms. J Clin Endocrinol Metab 2004;89:3813-7. http://dx.doi.org/10.1210/ic.2004-0143

22. Paduch DA, Brannigan RE, Fuchs EF, et al. The laboratory diagnosis of testosterone deficiency. Urology 2014;83:980-8. http://dx.doi.org/10.1016/i.urology.2013.12.024

23. Brambilla DJ, $O^{\prime}$ Donnell $A B$, Matsumoto $A M$, et al. Intraindividual variation in levels of serum testosterone and other reproductive and adrenal hormones in men. Clin Endocrinol (0xf) 2007;67:853-62. http:// dx.doi.org/10.1111/i.1365-2265.2007.02976.x

24. Vesper HW, Bhasin S, Wang $C$, et al. Interlaboratory comparison study of serum total testosterone [corrected] measurements performed by mass spectrometry methods. Steroids 2009;74:498-503. http:// dx.doi.org/10.1016/i.steroids.2009.01.004

25. Raff H, Sluss PM. Pre-analytical issues for testosterone and estradiol assays. Steroids 2008;73:1297-304. http://dx.doi.org/10.1016/i.steroids.2008.05.005

26. Nieschlag E, Behre HM, Bouchard $\mathrm{P}$, et al. Testosterone replacement therapy: Current trends and future directions. Hum Reprod Update 2004;10:409-19. http://dx.doi.org/10.1093/humupd/dmh035

27. Vigen $\mathrm{R}, \mathrm{O}^{\prime}$ Donnell $\mathrm{Cl}$, Baron $\mathrm{AE}$, et al. Association of testosterone therapy with mortality, myocardial infarction, and stroke in men with low testosterone levels. JAMA 2013;310:1829-36. http://dx.doi. org/10.1001/jama.2013.280386

28. LeBrasseur NK, Lajevardi N, Miciek R, et al. Effects of testosterone therapy on muscle performance and physical function in older men with mobility limitations (The TOM Trial): design and methods. Contemp Clin Trials 2009;30:133-40. http://dx.doi.org/10.1016/i.cct.2008.10.005

29. Finkle WD, Greenland S, Ridgeway GK, et al. Increased risk of non-fatal myocardial infarction following testosterone therapy prescription in men. PLoS One 2014;9:e85805. http://dx.doi.org/10.1371/ journal.pone.0085805

Correspondence: Dr. Ranith Ramasamy, 1 Baylor Plaza, Department of Urology, Baylor College of Medicine, Houston TX 77030; ranijthrama@gmail.com 